\title{
Uniform convergence of the Fourier series expansions in the subsystems of root functions of some spectral problem for ordinary differential equations of fourth order
}

\author{
Konul F. Abdullayeva *
}

Received: 28.08.2019 / Revised: 16.03.2020 / Accepted: 10.05.2020

\begin{abstract}
In this paper, we consider the spectral problem for ordinary differential equations of fourth order with a spectral parameter in the boundary conditions. We study the uniform convergence of expansions of continuous functions in the subsystem of root functions of this problem.
\end{abstract}

Keywords. eigenvalue problem, eigenvalue, root functions, Fourier series expansions

Mathematics Subject Classification (2010): 34B05, 34B08, 34B24, 34C23, 34L10, 34L15, 34L20

\section{Introduction}

We consider the following eigenvalue problem

$$
\begin{gathered}
(\ell y)(x) \equiv y^{(4)}(x)-\left(q(x) y^{\prime}(x)\right)^{\prime}=\lambda y(x), 0<x<1, \\
y^{\prime}(0) \cos \alpha-y^{\prime}(0) \sin \alpha=0, \\
y(0) \cos \beta+T y^{\prime}(0) \sin \beta=0, \\
y^{\prime}(1) \cos \gamma+y^{\prime \prime}(1) \sin \gamma=0, \\
(a \lambda+b) y(1)-(c \lambda+d) T y(1)=0,
\end{gathered}
$$

where $\lambda$ is a spectral parameter, $q(x) \in A C[0,1]$ and $q(x)>0$ for $x \in[0,1], T y \equiv$ $y^{\prime \prime \prime}-q y^{\prime}, \alpha, \beta, \gamma, a, b, c, d$ are real constants such that $\alpha, \beta, \gamma \in\left[0, \frac{\pi}{2}\right], c \neq 0$ and $\sigma=b c-a d \neq 0$.

The eigenvalue problems for ordinary differential equations with a spectral parameter in the boundary conditions arise in studies of various processes of mechanics and physics (see, e.g. $[7,11,12])$. From this point of view, research on these types of problems is relevant. Note that the spectral problems for ordinary differential equations of second and fourth orders with a spectral parameter in the boundary conditions in various settings have been studied by many authors (see [1-12, 15-23] and theirs bibliography).

Problem (1.1)-(1.5) has been considered in papers [3, 21], where the spectral properties of this problem were studied in detail. In [21] it was shown that the eigenvalues of the

\footnotetext{
* Corresponding author
}

Konul F. Abdullayeva

Sumgait State University, Sumgait AZ5008, Azerbaijan

E-mail: konul.abdullayeva.15@mail.ru 
spectral problem (1.1)-(1.5) with $\sigma>0$ are real, simple and form an unboundedly increasing sequence $\left\{\lambda_{k}\right\}_{k=1}^{\infty}$; here it was proved that the corresponding system of eigenfunctions $\left\{y_{k}\right\}_{k=1}^{\infty}$ forms a basis in the space $L_{p}, 1<p<\infty$, (for $p=2$ an unconditional basis) after removing an arbitrary fixed function. In the paper [3] it was proved that the eigenvalues $\lambda_{k}, k \in \mathbb{N}$, of (1.1)-(1.5) with $\sigma<0$ form an unbounded sequence without a finite limit point and either all eigenvalues are real and simple, or all eigenvalues are real and all but one double or triple are simple, or all eigenvalues are simple and all but one pair of non-real conjugates are real; in both cases $\lambda_{k}>0$ for $k \geq 3$. Moreover, in [3] it was established a necessary and sufficient condition for the system of root functions $\left\{y_{k}\right\}_{k=1}^{\infty}$ of problem (1.1)-(1.5) to form a basis in $L_{p}, 1<p<\infty$, (for $p=2$ an unconditional basis) after removing one function.

In studying boundary value problems for partial differential equations that reduce to eigenvalue problems for ordinary differential equations with a spectral parameter in the boundary conditions, an important role is played by the study of the uniform convergence of spectral expansions in the root functions of these spectral problems. The uniform convergence of Fourier series expansions of continuous functions in the subsystems of root functions of the second and fourth order Sturm-Liouville problem with a spectral parameter contained in the boundary conditions was considered in [7, 8, 15, 16, 18, 22, 23].

The purpose of this paper is to establish conditions under which the spectral expansions of continuous functions in the subsystem of root functions of problem (1.1)-(1.5) are convergent uniformly.

\section{Preliminary}

As is known [3], problem (1.1)-(1.5) reduces to the eigenvalue problem for the linear operator $L$ in the Hilbert space $H=L_{2}(0, l) \oplus \mathbb{C}$ with the scalar product

$$
(\widehat{y}, \widehat{v})=(\{y(x), m\},\{v(x), s\})=\int_{0}^{1} y(x) \overline{v(x)} d x+\sigma^{-1} m \bar{s},
$$

where the operator $L$ defined by

$$
L \widehat{u}=\{\ell y(x), d T y(1)-b y(1)\}
$$

in the domain

$$
\begin{gathered}
D(L)=\left\{\widehat{u}=\{y(x), m\} \in H: y \in W_{2}^{4}(0,1), \ell y \in L_{2}(0,1),\right. \\
\left.y(0)=y^{\prime}(0)=y^{\prime \prime}(1)=0, m=a y(1)-c T y(1)\right\} .
\end{gathered}
$$

In this case, the problem (1.1)-(1.5) is rewritten in the following operator equation

$$
L \widehat{y}=\lambda \widehat{y}, \widehat{y} \in D(L) .
$$

Hence the eigenvalues $\lambda_{k}, k \in \mathbb{N}$, of the operator $L$ and problem (1.1)-(1.5) coincide taking into account their multiplicity, and between the root vectors there, is a one-to-one correspondence

$$
\widehat{y}_{k} \longleftrightarrow\left\{y_{k}, m_{k}\right\}, m_{k}=a y_{k}(1)-c T y_{k}(1) .
$$

In the case $\sigma>0$ operator $L$ is a self-adjoint lower-semibounded operator in $H$. In the case $\sigma<0$ this operator is closed (nonself-adjoint) in $H$ and has a compact resolvent.

We define number $\varkappa$ as follows:

$$
\varkappa= \begin{cases}0, & \text { if } \sigma>0 \\ 1, & \text { if } \sigma<0\end{cases}
$$


Then the system of eigenvectors $\left\{y_{k}(x), m_{k}\right\}_{k=1}^{\infty}$ forms an orthogonal basis in $H$. Let $J$ : $H \rightarrow H$ be the operator given by

$$
J\{y, m\}=\{y,-m \operatorname{sgn} \sigma\} .
$$

It is obvious that this operator is unitary and symmetric on $H$, and its spectrum in the case $\sigma>0$ consists of one eigenvalue: +1 with infinite multiplicity; in the case of $\sigma<0$ consists of two eigenvalues: -1 with multiplicity 1 and +1 with infinite multiplicity. Hence operator $J$ generates the Pontryagin space $\Pi_{\varkappa}$ with $J$-metric

$$
[\widehat{y}, \widehat{v}]=[\{y(x), m\},\{v(x), s\}]=\int_{0}^{1} y(x) \overline{v(x)} d x+(-1)^{\varkappa} \sigma^{-1} m \bar{s} .
$$

Note that if $\sigma>0$, then $\Pi_{\varkappa}=H$ and $J=I$, where $I: H \rightarrow H$ is identity operator.

Theorem 2.1 [3, Theorems 2.1, 6.1 and Lemma 2.1] The following statements hold:

(i) the operator $L$ is $J$-self-adjoint in $\Pi_{\varkappa}$;

(ii) if $L^{*}$ is an adjoint operator of $L$ in $H$, then $L^{*}=J L J$;

(iii) the system of root vectors $\left\{\widehat{y}_{k}\right\}_{k=1}^{\infty}$ of the operator L forms a Riesz basis in $H$.

Let $\widehat{v}_{k}=\left\{v_{k}(x), s_{k}\right\}$ be the system adjoint to the system $\left\{\widehat{y}_{k}\right\}_{k=1}^{\infty}$ that is given by the relation (6.5) of [3].

Theorem 2.2 [21, Theorem 5.1] and [3, Theorem 6.2] Let $r$ be an arbitrary fixed natural number. If $\sigma>0$, then the system $\left\{y_{k}\right\}_{k=1, k \neq r}^{\infty}$ is a basis in the space $L_{p}(0,1), 1<p<\infty$, (for $p=2$ this basis is an unconditional basis); if $\sigma<0$, then the system $\left\{y_{k}\right\}_{k=1, k \neq r}^{\infty}$ is a basis in $L_{p}(0,1), 1<p<\infty$ (for $p=2$ this basis is an unconditional basis) if and only if $s_{r} \neq 0$; in this case the system $\left\{u_{k}(x)\right\}_{k=1, k \neq r}^{\infty}, u_{k}(x)=v_{k}(x)-s_{k} s_{r}^{-1} v_{r}(x)$, is adjoint to the system $\left\{y_{k}\right\}_{k=1, k \neq r}^{\infty}$.

Remark 2.1 The conditions under which $s_{r} \neq 0$ are given in [3, Corollary 6.1]; in particular, if $\lambda_{r}$ is a simple eigenvalue of problem (1.1)-(1.5), then $s_{r} \neq 0$.

In the case $\sigma>0$ let $r$ be an arbitrary fixed natural number, in the case $\sigma<0$ let $r$ be an arbitrary fixed natural number such that $s_{r} \neq 0$. Then by virtue of Theorem 2.2 any function $f(x) \in C[0,1]$ is expanded in the Fourier series

$$
f(x)=\sum_{k=1}^{\infty}\left(f, u_{k}\right) y_{k}(x),
$$

in the system $\left\{y_{k}\right\}_{k=1, k \neq r}^{\infty}$ which converges in $L_{p}(0,1), 1<p<\infty$, and for $p=2$ this series converges unconditionally.

To study the uniform convergence on $[0,1]$ of the Fourier series (2.1) of function $f(x) \in$ $C[0,1]$ in the system of root functions $\left\{y_{k}\right\}_{k=1, k \neq r}^{\infty}$ of problem (1.1)-(1.5) we establish a correspondence between the root functions of this problem and the problem (1.1)-(1.4),

$$
a y(1)-c T y(1)=0,
$$

with $q(x) \equiv 0$ (the eigenvalues of which are real, simple and form an infinitely increasing sequence $\left\{\mu_{k}\right\}_{k=1}^{\infty}$ such that $\mu_{k}>0$ for $k \geq 2$; moreover, the eigenfunction $\vartheta_{k}(x), k \geq 2$, corresponding to the eigenvalue $\mu_{k}$ has $k-1$ simple zeros in $(0,1)$ (see $\left.[10,16,18]\right)$ ). The establishment of such a correspondence is based on the use of more refined asymptotic formulas for the eigenvalues and eigenfunctions of these problems which are obtained in [21, Theorem 3.1] and [3, Theorem 5.1] (see also [20, Theorem 6.1]). It should be noted that 
in the case of $\alpha=0, \beta=0$ and $\gamma=\frac{\pi}{2}$, refined asymptotic formulas for eigenvalues and eigenfunctions of problems (1.1)-(1.4), (2.2) with $q(x) \equiv 0$ and (1.1)-(1.5) were obtained in the recent paper [1]. Using these asymptotic formulas we establish the necessary correspondence and find sufficient conditions for the uniform convergence on $[0,1]$ of the series (2.1).

\section{Uniform convergence of the series (2.1)}

In this section we will consider the case when $\alpha=0, \beta=0$ and $\gamma=\frac{\pi}{2}$ in the boundary conditions (1.2)-(1.4). It should be noted that the remaining cases are treated similarly.

Theorem 3.1 Let $r$ be an arbitrarily fixed positive integer (moreover, $s_{r} \neq 0$ for $\sigma<0$ ), $f(x)$ be the continuous function on $[0,1]$ and the Fourier series expansion

$$
f(x)=\sum_{k=1}^{\infty}\left(f, \vartheta_{k}\right) \vartheta_{k}(x)
$$

of this function in the system $\left\{\vartheta_{k}(x)\right\}_{k=1}^{\infty}, \vartheta_{k}(x)=\left\|\phi_{k}\right\|_{2}^{-1} \phi_{k}(x)$ is convergent uniformly on $[0,1]$, where $\|\cdot\|_{2}$ is the norm in the space $L_{2}(0,1)$. If $\left(f, v_{r}\right)=0$ or $\left(f, v_{r}\right) \neq 0$ and $d=0$, then the series (2.1) uniformly converges on the interval $[0,1]$; if $\left(f, v_{r}\right) \neq 0$ and $d \neq 0$, then the series (2.1) uniformly converges on the interval $[0, \tau]$ for any $0<\tau<1$.

Proof. Since $\alpha=0, \beta=0$ and $\gamma=\frac{\pi}{2}$ in boundary conditions (1.2)-(1.4) it follows from [1, Theorem 3.1 and 4.1$]$ that

$$
\begin{gathered}
\sqrt[4]{\mu_{k}}=\left(k-\frac{1}{2}\right) \pi+O\left(\frac{1}{k^{3}}\right) \\
\phi_{k}(x)=\sin \left(k-\frac{1}{2}\right) \pi x-\cos \left(k-\frac{1}{2}\right) \pi x+e^{-\left(k-\frac{1}{2}\right) \pi x} \\
+(-1)^{k+1} e^{\left(k-\frac{1}{2}\right) \pi(x-1)}+O\left(\frac{1}{k^{3}}\right), \\
\sqrt[4]{\lambda_{k}}=\left(k-\frac{3}{2}\right) \pi+\frac{q_{0}}{4 k \pi}+O\left(\frac{1}{k^{2}}\right), \\
y_{k}(x)=\sin \left(k-\frac{3}{2}\right) \pi x-\cos \left(k-\frac{3}{2}\right) \pi x+e^{-\left(k-\frac{3}{2}\right) \pi x}+(-1)^{k} e^{\left(k-\frac{3}{2}\right) \pi(x-1)} \\
+\frac{q_{0} x-q_{0}(x)}{4 k \pi}\left\{\sin \left(k-\frac{3}{2}\right) \pi x+\cos \left(k-\frac{3}{2}\right) \pi x\right\}-\frac{q_{0} x+q_{0}(x)}{4 k \pi} e^{-\left(k-\frac{3}{2}\right) \pi x} \\
+(-1)^{k} \frac{q_{0} x-q_{1}(x)-q_{0}}{4 k \pi} e^{\left(k-\frac{3}{2}\right) \pi(x-1)}+O\left(\frac{1}{k^{2}}\right),
\end{gathered}
$$

where

$$
q_{0}(x)=\int_{0}^{x} q(t) d t, q_{0}=\int_{0}^{1} q(x) d x, q_{1}(x)=\int_{x}^{1} q(t) d t,
$$

and the relations (3.3) and (3.3) hold uniformly for $x \in[0,1]$.

By following the arguments in Theorem 4.1 of [1] we obtain the following asymptotic formulas 


$$
\begin{aligned}
& y_{k}^{\prime}(x)=\left(k-\frac{3}{2}\right) \pi\left\{\cos \left(k-\frac{3}{2}\right) \pi x+\sin \left(k-\frac{3}{2}\right) \pi x-e^{-\left(k-\frac{3}{2}\right) \pi x}\right. \\
& +(-1)^{k} e^{\left(k-\frac{3}{2}\right) \pi(x-1)}+\frac{q_{0} x-q_{0}(x)}{4 k \pi}\left\{\cos \left(k-\frac{3}{2}\right) \pi x-\sin \left(k-\frac{3}{2}\right) \pi x\right\} \\
& \left.+\frac{q_{0} x+q_{0}(x)}{4 k \pi} e^{-\left(k-\frac{3}{2}\right) \pi x}+(-1)^{k} \frac{q_{0} x-q_{1}(x)-q_{0}}{4 k \pi} e^{\left(k-\frac{3}{2}\right) \pi(x-1)}+O\left(\frac{1}{k^{2}}\right)\right\}, \\
& y_{k}^{\prime \prime \prime}(x)=-\left(k-\frac{3}{2}\right)^{3} \pi^{3}\left\{\cos \left(k-\frac{3}{2}\right) \pi x+\sin \left(k-\frac{3}{2}\right) \pi x+e^{-\left(k-\frac{3}{2}\right) \pi x}\right. \\
& +(-1)^{k} e^{\left(k-\frac{3}{2}\right) \pi(x-1)}+\frac{q_{0} x-q_{0}(x)}{4 k \pi}\left\{\cos \left(k-\frac{3}{2}\right) \pi x-\sin \left(k-\frac{3}{2}\right) \pi x\right\} \\
& \left.-\frac{q_{0} x+q_{0}(x)}{4 k \pi} e^{-\left(k-\frac{3}{2}\right) \pi x}-(-1)^{k} \frac{q_{0} x-q_{1}(x)-q_{0}}{4 k \pi} e^{\left(k-\frac{3}{2}\right) \pi(x-1)}+O\left(\frac{1}{k^{2}}\right)\right\} .
\end{aligned}
$$

It follows from formulas (3.5)-(3.7) that

$$
\begin{gathered}
y_{k}(1)=2(-1)^{k}+O\left(\frac{1}{k^{2}}\right), y_{k}^{\prime}(1)=\left(k-\frac{3}{2}\right) \pi\left\{2(-1)^{k}+O\left(\frac{1}{k^{2}}\right)\right\}, \\
y_{k}^{\prime \prime \prime}(1)=\left(k-\frac{3}{2}\right)^{3} \pi^{3}\left\{2(-1)^{k+1}+O\left(\frac{1}{k^{2}}\right)\right\} .
\end{gathered}
$$

Then, by (3.4) and (3.8) we get

$$
\begin{gathered}
m_{k}=a y_{k}(1)-c T y_{k}(1)=\frac{d T y_{k}(1)-b y_{k}(1)}{\lambda_{k}} \\
=\frac{d\left(y_{k}^{\prime \prime \prime}(1)-q(1) y_{k}^{\prime}(1)\right)-b y_{k}(1)}{\lambda_{k}}=\left\{\begin{array}{cl}
O\left(\frac{1}{k^{4}}\right), & \text { if } d=0, \\
\frac{2 d(-1)^{k+1}}{k}\left(1+O\left(\frac{1}{k^{2}}\right)\right), & \text { if } d \neq 0 .
\end{array}\right.
\end{gathered}
$$

Let $r^{*}=r$ if $\sigma>0, r^{*}=\max \{3, r, M+2\}$ if $\sigma<0$ (for determining the number $M$ see [3, Theorem 4.1; pp 771-773]). Note that if $k \geq r^{*}$, then it follows from [20, Theorem 5.1] and [3, Theorem 4.1] that $\varrho\left(\lambda_{k}\right)=1$, and consequently, by Theorem 7.1 of [20] and Lemmas $6.1,6.2$ and 6.3 of [3] we have

$$
\begin{gathered}
v_{k}=\delta_{k}^{-1} y_{k}, s_{k}=\delta_{k}^{-1} m_{k}=\delta_{k}^{-1}\left(a y_{k}(1)-c T y_{k}(1)\right), \\
u_{k}(x)=v_{k}(x)-s_{k} s_{r}^{-1} v_{r}(x),
\end{gathered}
$$

where

$$
\delta_{k}=\left\|y_{k}\right\|_{2}^{2}+\sigma^{-1} m_{k}^{2}
$$

From (3.3) and (3.5) by direct calculations we obtain the following relations

$$
\left\|y_{k}\right\|_{2}^{2}=1+O\left(\frac{1}{k^{2}}\right),\left\|\phi_{k}\right\|_{2}^{2}=1+O\left(\frac{1}{k^{3}}\right) .
$$

Moreover, by (3.9) and (3.12) from (3.11) we get

$$
\delta_{k}=1+O\left(\frac{1}{k^{2}}\right) .
$$

By virtue of Theorem 2.2, (3.12) and (3.13) it follows from (3.10) that

$$
v_{k}=y_{k}\left(1+O\left(\frac{1}{k^{2}}\right)\right), s_{k}=\left\{\begin{array}{cl}
O\left(\frac{1}{k^{4}}\right), & \text { if } d=0, \\
\frac{2 d(-1)^{k+1}}{k}\left(1+O\left(\frac{1}{k^{2}}\right)\right), & \text { if } d \neq 0 .
\end{array}\right.
$$


Obviously, to prove the uniform convergence on $[0,1]$ of the series $(2.1)$, it suffices to show that the following series converges uniformly on $[0,1]$ :

$$
\widetilde{f}(x)=\sum_{k=r^{*}+1}^{\infty}\left(f, u_{k}\right) y_{k}(x) .
$$

Then, by the first relation in (3.14), it follows from (3.15) that

$$
f_{1}(x)=\sum_{k=r^{*}+1}^{\infty}\left(f, y_{k}\right) y_{k}(x)-s_{r}^{-1}\left(f, v_{r}\right) \sum_{k=r^{*}+1}^{\infty} s_{k} y_{k}(x)+\sum_{k=r^{*}+1}^{\infty} O\left(\frac{1}{k^{2}}\right) .
$$

From the asymptotic formulas (3.3) and (3.12) we see that

$$
\begin{aligned}
\vartheta_{k}(x)= & \sin \left(k-\frac{1}{2}\right) \pi x-\cos \left(k-\frac{1}{2}\right) \pi x+e^{-\left(k-\frac{1}{2}\right) \pi x} \\
& +(-1)^{k+1} e^{\left(k-\frac{1}{2}\right) \pi(x-1)}+O\left(\frac{1}{k^{3}}\right) .
\end{aligned}
$$

Consequently, by (3.5) and (3.17) we have

$$
y_{k}(x)=\vartheta_{k-1}(x)+O\left(\frac{1}{k}\right) .
$$

Then, by (3.18), it follows from (3.16) that

$$
\begin{aligned}
& \widetilde{f}(x)=\sum_{k=r^{*}+1}^{\infty}\left(f, y_{k}\right) v_{k-1}(x)+\sum_{k=r^{*}+1}^{\infty}\left(f, y_{k}\right) O\left(\frac{1}{k}\right) \\
& -s_{r}^{-1}\left(f, v_{r}\right)\left(\sum_{k=r^{*}+1}^{\infty} s_{k} \vartheta_{k-1}(x)+\sum_{k=r^{*}+1}^{\infty} s_{k} O\left(\frac{1}{k}\right)\right) .
\end{aligned}
$$

In view of Theorem 2.2 we get

$$
\left|\sum_{k=r^{*}+1}^{\infty}\left(f, y_{k}\right) O\left(\frac{1}{k}\right)\right| \leq \operatorname{const}\left(\sum_{k=r^{*}+1}^{\infty}\left|\left(f, y_{k}\right)\right|^{2}+\sum_{k=r+3}^{\infty} \frac{1}{k^{2}}\right)^{\frac{1}{2}} .
$$

Moreover, by (3.14) we have

$$
\sum_{k=r^{*}+1}^{\infty} s_{k} O\left(\frac{1}{k}\right)=\sum_{k=r^{*}+1}^{\infty} O\left(\frac{1}{k^{2}}\right) .
$$

Thus, to study the uniform convergence of the series (3.15) it is enough to study the uniform convergence of series:

$$
\begin{gathered}
\sum_{k=r^{*}+1}^{\infty}\left(f, y_{k}\right) \vartheta_{k-1}(x), \\
\sum_{k=r^{*}+1}^{\infty} s_{k} \vartheta_{k-1}(x) .
\end{gathered}
$$

The functions $g_{1}(x), g_{2}(x), g_{3}(x)$ and $g_{4}(x), x \in[0,1]$, we define by

$$
g_{1}(x) \equiv g_{2}(x)=\frac{q_{0} x-q_{0}(x)}{4 \pi}, g_{3}(x)=\frac{q_{0} x+q_{0}(x)}{4 \pi}, g_{4}(x)=\frac{q_{0} x-q_{1}(x)-q_{0}}{4 \pi} \text {. }
$$

Then by (3.3) and (3.5) we have 


$$
\begin{gathered}
y_{k}(x)=\vartheta_{k-1}(x)+\frac{p_{1}(x)}{k} \sin \left(k-\frac{3}{2}\right) \pi x+\frac{p_{2}(x)}{k} \cos \left(k-\frac{3}{2}\right) \pi x- \\
-\frac{p_{3}(x)}{k} e^{-\left(k-\frac{3}{2}\right) \pi x}+(-1)^{k} \frac{p_{4}(x)}{k} e^{\left(k-\frac{3}{2}\right) \pi(x-1)}+O\left(\frac{1}{k^{2}}\right) .
\end{gathered}
$$

Consequently, the series (3.20) has the form

$$
\begin{gathered}
\sum_{k=r^{*}+1}^{\infty}\left(f, y_{k}\right) \vartheta_{k-1}(x)=\sum_{k=r^{*}+1}^{\infty}\left(f, \vartheta_{k-1}\right) \vartheta_{k-1}(x)+\sum_{k=r^{*}+1}^{\infty} \frac{\left(f g_{1}, \epsilon_{k}^{(1)}\right)}{k} \vartheta_{k-1}(x) \\
+\sum_{k=r^{*}+1}^{\infty} \frac{\left(f g_{2}, \epsilon_{k}^{(2)}\right)}{k} \vartheta_{k-1}(x)-\sum_{k=r^{*}+1}^{\infty} \frac{\left(f g_{3}, \epsilon_{k}^{(3)}\right)}{k} \vartheta_{k-1}(x)+\sum_{k=r^{*}+1}^{\infty} \frac{\left(f g_{4}, \epsilon_{k}^{4}\right)}{k} \vartheta_{k-1}(x) \\
+\sum_{k=r^{*}+1}^{\infty} \vartheta_{k-1}(x) O\left(\frac{1}{k^{2}}\right) .
\end{gathered}
$$

where

$$
\begin{aligned}
\epsilon_{k}^{(1)}(x)=\sin \left(k-\frac{3}{2}\right) \pi x, \epsilon_{k}^{(2)}(x) & =\cos \left(k-\frac{3}{2}\right) \pi x, \epsilon_{k}^{(3)}(x)=e^{-\left(k-\frac{3}{2}\right) \pi x}, \\
\epsilon_{k}^{(4)}(x) & =e^{\left(k-\frac{3}{2}\right) \pi(x-1)},
\end{aligned}
$$

which are Bessel systems in view of Lemma 5 of [24]. Due to the Besslessness of these systems, we have

$$
\left|\sum_{k=r^{*}+1}^{\infty} \frac{\left(f g_{i}, \epsilon_{k}^{(i)}\right)}{k}\right| \leq C_{1}\left(\sum_{k=r^{*}+1}^{\infty} \frac{1}{k^{2}}+\sum_{k=r^{*}+1}^{\infty}\left|\left(f g_{i}, \epsilon_{k}^{(i)}\right)\right|^{2}\right) \leq C_{2}\left(1+\|f\|_{2}^{2}\right), i=\overline{1,4},
$$

where $C_{1}$ and $C_{2}$ some positive constants. Then it follows that the series (3.20) uniformly converges on $[0,1]$, because the series (3.1) uniformly converges on $[0,1]$.

Let $\left(f, v_{r}\right)=0$. Then based on the uniform convergence of the series $(3.20)$ on $[0,1]$ from (3.19) we obtain that the series (2.1) converges uniformly on $[0,1]$.

Let $\left(f, v_{r}\right) \neq 0$ and $d=0$ in the boundary condition (1.5). Then by the second relation in (3.14) we have $s_{k}=O\left(\frac{1}{k^{4}}\right)$. Hence it follows that the series (3.21) uniformly converges on $[0,1]$, and consequently, the series $(2.1)$ uniformly converges on $[0,1]$.

Now let $\left(f, v_{r}\right) \neq 0$ and $d \neq 0$ in the boundary condition (1.5). Hence it follows from the second relation in (3.14) that $s_{k}=\frac{2 d(-1)^{k+1}}{k}\left(1+O\left(\frac{1}{k^{2}}\right)\right)$. Then the series (3.21) has the form

$$
\sum_{k=r^{*}+1}^{\infty} s_{k} \vartheta_{k-1}(x)=2 d \sum_{k=r^{*}+1}^{\infty} \frac{(-1)^{k+1}}{k} \vartheta_{k-1}(x)+\sum_{k=r^{*}+1}^{\infty} O\left(\frac{1}{k^{2}}\right) .
$$

By following the arguments in Theorem 8.1 of [8, pp. 300-301] the series

$$
\sum_{k=r^{*}+1}^{\infty} \frac{(-1)^{k+1}}{k} \vartheta_{k-1}(x)
$$

does not converge uniformly on the interval $[0,1]$, but converges uniformly on the interval $[0, \tau]$ for any $0<\tau<1$. Consequently, the series (2.1) is convergent uniformly on the interval $[0, \tau]$ for any $0<\tau<1$. The proof of this theorem is complete. 


\section{References}

1. Abdullayeva, K.F.: Asymptotic formulas for eigenvalues and eigenfunctions of some ordinary differential operators of fourth order, Trans. Natl. Acad. Sci. Azerb. Ser. Phys.-Tech. Math. Sci. Mathematics, 38 (4), 8-16 (2018).

2. Aliyev, Z.S.: Basis properties of a fourth order differential operator with spectral parameter in the boundary condition, Cent. Eur. J. Math. 8 (2), 378-388 (2010).

3. Aliev, Z.S.: Basis properties in $L_{p}$ of systems of root functions of a spectral problem with spectral parameter in a boundary condition, Differ. Equ. 47 (6), $766-777$ (2011).

4. Aliev, Z.S.: On basis properties of root functions of a boundary value problem containing a spectral parameter in the boundary conditions, Doklady Math. 87 (2), 137-139 (2013).

5. Aliev, Z.S., Dunyamalieva, A.A.: Defect basis property of a system of root functions of a Sturm-Liouville problem with spectral parameter in the boundary conditions, Differ. Equ. 51 (10), 1249-1266 (2015).

6. Aliyev, Z.S., Guliyeva, S.B.: Properties of natural frequencies and harmonic bending vibrations of a rod at one end of which is concentrated inertial load, J. Differential Equations 263 (9), 5830-5845 (2017).

7. Aliyev, Z.S., Kerimov, N.B., Mehrabov, V.A.: Convergence of eigenfunction expansions for a boundary value problem with spectral parameter in the boundary conditions. I, Differ. Equ. 56 (2), 143-157 (2020).

8. Aliyev, Z.S., Kerimov, N.B., Mehrabov, V.A.: Convergence of eigenfunction expansions for a boundary value problem with spectral parameter in the boundary conditions. II, Differ. Equ. 56 (3), 291-302 (2020).

9. Aliyev, Z.S., Mamedova, G.T.: Some properties of eigenfunctions for the equation of vibrating beam with a spectral parameter in the boundary conditions, J. Differential Equations, (2020), doi.org/10.1016/j.jde.2020.01.010.

10. Aliyev, Z.S., Namazov, F.M.: Spectral properties of a fourth-order eigenvalue problem with spectral parameter in the boundary conditions, Electron. J. Differ. Equ. 2017 (307), 1-11 (2017).

11. Aliyev, Z.S., Namazov, F.M.: On the spectral problem arising in the mathematical model of bending vibrations of a homogeneous rod, Complex Anal. Oper. Theory, $\mathbf{1 3}$ (8), 3675-3693 (2019).

12. Aliyev, Z.S., Namazov, F.M.: Spectral properties of the equation of a vibrating rod at both ends of which the masses are concentrated, Banach J. Math. Anal. (2020), doi.org/10.1007/s43037-019-00009-1.

13. Banks, D.O., Kurowski, G.J.: A Prufer transformation for the equation of a vibrating beam subject to axial forces, J. Differential Equations, 24, 57-74 (1977).

14. Bolotin, B.B.: Vibrations in technique: Handbook in 6 volumes, The vibrations of linear systems, I, Engineering Industry, Moscow, 1978.

15. Kapustin, N.Yu.: On the uniform convergence in $C^{1}$ of Fourier series for a spectral problem with squared spectral parameter in a boundary condition, Differ. Equ. 47 (10), 1394-1399 (2011).

16. Kapustin, N.Yu., Moiseev, E.I.: Convergence of spectral expansions for functions of the Hölder class for two problems with a spectral parameter in the boundary condition, Differ. Equ. 36 (8), 1069-1074 (2001).

17. Kapustin, N.Yu., Moiseev, E.I.: On the basis property in the space $L_{p}$ of systems of eigenfunctions corresponding to two problems with spectral parameter in the boundary condition, Differ. Equ., 36 (10), 1357-1360 (2000).

18. Kapustin, N.Yu., Moiseev, E.I.: A remark on the convergence problem for spectral expansions corresponding to a classical problem with spectral parameter in the boundary condition, Differ. Equ. 37 (2), 1677-1683 (2001). 
19. Kerimov, N.B., Z.S. Aliev, Z.S.: On oscillation properties of the eigenfunctions of a fourth order differential operator, Trans. Natl. Acad. Sci. Azerb. Ser. Phys.-Tech. Math. Sci. 25 (4), 63-76 (2005).

20. Kerimov, N.B., Z.S. Aliev, Z.S.: Basis properties of a spectral problem with spectral parameter in the boundary condition, Sbornik: Mathematics, 197 (10), 1467-1487 (2006).

21. Kerimov, N.B., Z.S. Aliev, Z.S.: On the basis property of the system of eigenfunctions of a spectral problem with spectral parameter in a boundary condition, Differ. Equ. $\mathbf{4 3}$ (7), 905-915 (2007).

22. Kerimov, N.B., Goktas, S., Maris, E.A.: Uniform convergence of the spectral expansions in terms of root functions for a spectral problem, Electron. J. Differential Equations, 2016 (80), 1-14 (2016).

23. Kerimov, N.B., Maris, E.A.: On the uniform convergence of the Fourier Series for one spectral problem with a spectral parameter in a boundary condition, Math. Methods Appl. Sci. 39 (9), 2298-2309 (2016).

24. Keselman, G.M.: On the unconditional convergence of eigenfunction expansions of certain differential operators, Izv. Vyssh. Uchebn. Zaved. Mat. 39 (2), $82-93$ (1964) [in Russian]. 